\title{
Forecasting-Based Adaptive Optimized Dispatch in Smart Grid Online
}

\author{
Qiang Jiang1, Dan Hu${ }^{1}$, Duyi He ${ }^{2}$ \\ ${ }^{1}$ School of Physics and Electronic Engineering, Leshan Normal University, Leshan, China \\ ${ }^{2}$ Moro Library, Leshan Normal University, Leshan, China \\ Email: Qiangjianglsnu@outlook.com, hudan@lsnu.edu.cn, ruihe0406@163.com
}

How to cite this paper: Jiang, Q., Hu, D. and He, D.Y. (2020) Forecasting-Based Adaptive Optimized Dispatch in Smart Grid Online. International Journal of Modern Nonlinear Theory and Application, 9, 1-18.

https://doi.org/10.4236/ijmnta.2020.91001

Received: November 17, 2019

Accepted: February 7, 2020

Published: February 10, 2020

Copyright $\odot 2020$ by author(s) and Scientific Research Publishing Inc. This work is licensed under the Creative Commons Attribution International License (CC BY 4.0).

http://creativecommons.org/licenses/by/4.0/

\begin{abstract}
The power grid is a fusion of technologies in energy systems, and how to adjust and control the output power of each generator to balance the load of the grid is a crucial issue. As a platform, the smart grid is for the convenience of the implementation of adaptive control generators using advanced technologies. In this paper, we are introducing a new approach, the Central Lower Configuration Table, which optimizes dispatch of the generating capacity in a smart grid power system. The dispatch strategy of each generator in the grid is presented in the configuration table, and the scenario consists of two-level agents. A central agent optimizes dispatch calculation to get the configuration table, and a lower agent controls generators according to the tasks of the central level and the work states during generation. The central level is major optimization and adjustment. We used machine learning to predict the power load and address the best optimize cost function to deal with a different control strategy. We designed the items of the cost function, such as operations, maintenances and the effects on the environment. Then, according to the total cost, we got a new second-rank-sort table. As a result, we can resolve generator's task based on the table, which can also be updated on-line based on the environmental situation. The signs of the driving generator's controller include active power and system's $f$. The lower control level agent carries out the generator control to track $f$ along with the best optimized cost function. Our approach makes optimized dispatch algorithm more convenient to realize, and the numerical simulation indicates the strategy of machine learning forecast of optimized power dispatch is effective.
\end{abstract}

\section{Keywords}

Machine Learning, Smart Grid, Adaptive Control, Dispatch Power, Online 


\section{Introduction}

With the fast society innovation speed, the higher demand for power industry is required, such as higher quality, better stability, lower harmonics and inter-harmonics, etc. Therefore, it has become inexorable trend that utilizing modern science technology improves power system quality, and smart grid will and must be the future electric system [1] [2]. In [1], the characteristics of the smart grid are to change the conventional concept of energy management and operation, optimise the automation of the electricity flow through the system, reduce losses and costs of energy, and improve reliability and economy. In [3], smart management is the main content in smart grid, which focuses on energy efficiency and demands profile improvement, such as cost optimization, price stabilization and emission control. In a power grid system, various generations technologies function together, such as wind energy, solar PV (photovoltaic) energy, hydropower, nuclear energy, coal fired power, etc., and they all have different characters, for example the wind and PV energy are fluctuated and stochastic. In addition to that, there are lots of power supply nodes (electrical generations) and too many demand nodes (electrical consumers) in a power grid, which makes more difficulties to realize high quality supply electric energy, shown in Figure 1.

There are two kinds of thoughts to implement control; one is adjusting the side of demand via electricity price. In recent years, researchers have got some results. I. Dusparic et al., in [4], in order to optimize energy usage, proposed a multi-agent approach. The main demand is household load, which uses load forecasting response demand, by reinforcement learning agent controlling household electrical devices, and uses current load and 24 hours forecast load to shift peak demand to an off-peak period to implement demand response. In [5], Z. Wen et al. addressed a novel energy management system (EMS) formulation for demand response problem in the residential and other small users. The EMS does not require a disutility function that can eliminate impractical assumption,

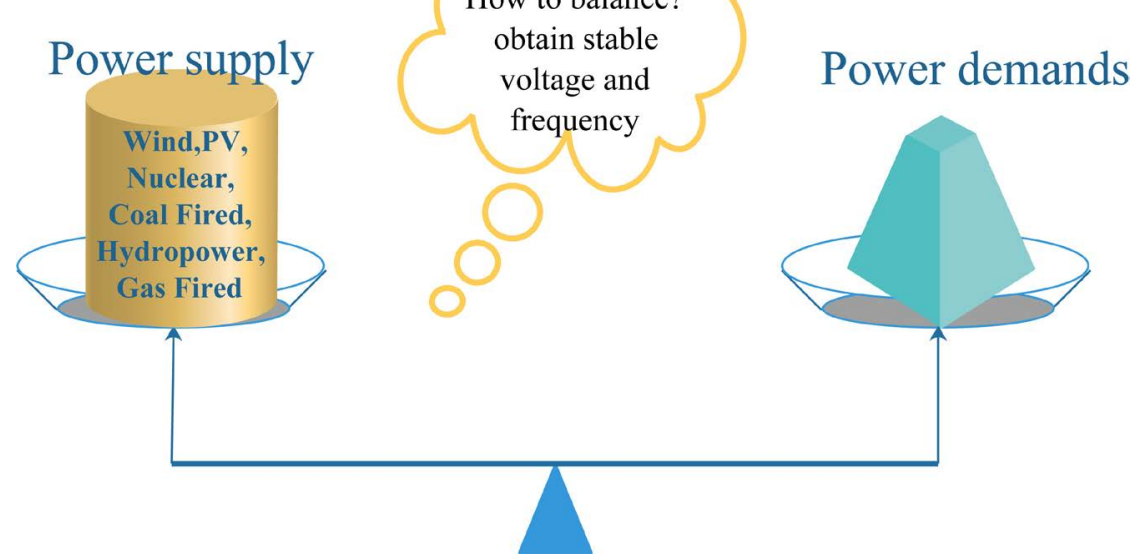

Figure 1. Energy structure of electric power. 
and allows both users initiated jobs and EMS initiated jobs, more flexible requests. K. Khezeli et al. issued risk sensitive learning and electrical pricing for demand response [6], and so on. Above all, demand response research results are reducing demand fluctuation, making the load curve more smoothness and improving forecasting accuracy.

The other way is controlling the side of supply (include generators, optimize adjustment system). Many researchers focus on the side of generation [7]-[15]. [7] proposed a wireless-coded power control approach for double-feed-induction wind generators. This system uses adaptive neuro fuzzy control method to improve the system robustness. In recent years, the state of research on photovoltaic electricity generator dynamic modeling ways is reviewed, such as different classifications for PV cell modeling methods, piecewise linear circuit and analytical and numerical techniques, etc. [13]. In [11], I. Jayawardene and G.K. Venayagamoorthy presented an approach that predicts the output of PV power plant use current weather parameters, named adaptive neuro-fuzzy inference system (ANFIS), and the predicted accuracy MAPE (mean absolute percentage error) is 25.8 .

In adjustment and electrical power system optimization aspect, some methods are published [8] [9] [12] [14] [16] [17] [18] [19]. In [8], research a smart control strategy to dample power system stabilizer (PSS), apply ANFIS technology obtained very good dampling of PSS and almost eliminates the oscillations in the power system. In [9], the authors used smart energy storage system module to reduce the fluctuation of wind energy system, used ANN for wind forecast, and used improved bacterial foraging algorithm to realise multi-objective optimization, consider some conditions, such as operating cost, net emission, pollutions, electrical power balance. Ref. [12] proposed a novel neuro-controller into the multi-machine power system and provided an adaptive supplemental control signal for each exciter to deal with the speed difference each generator. Refs. [14] [16] [17] [18] [19], present optimization for energy system. DeSantis posed genetic algorithm for power flow management in a micro-grid, and use genetic algorithm adaptive tune the rules to the suitable system. O. Nadjemi et al. utility cuckoo search algorithm to optimize hybrid PV and wind energy system sizing. F. Almonacid et al. review of techniques on neural networks for photovoltaic technology. W.L. Theo et al. review the distributed generation system planning and optimisation techniques.

Related works in recent years some researches were published [20]-[29]. In [20], a kind of scenario that maximizes social welfare for the question of transmission losses, and a consensus-based algorithm was designed and proved its convergence and optimality. In [21], presented a routing economic dispatch algorithm, the main goal is to deduce the transmission loss by optimizing the rout of power flow. Reference [22] described a protocol, which is robust mixed weather forecasts to improve the performance of a hybrid microgrid and reducing costs. In [23], a decentralized approach for economic dispatch in smart grid was proposed, which is performed based on a distributed multi-agent system. 
The protocol is comprised of two strategies, consesus strategy and threshold strategy, the former is due to adaptive updating rules and the latter works for frequency regulation. In [25], proposed a multi-agent optimal microgrid control strategy in order to realise distributed energy resources economic dispatch. The lower agent performs frequency adjustment on line, and the upper level implements distributed communication between agents for microgrid management. Above all, many results focus on research demands or generators control, but how to optimize control different technology generations become the new problem, especially we are in the times of large-scale power grid. In this paper, we will research how to optimize controlling all classes of technologies energy in a power system based on the optimal cost. We try our best to find a method to perform the optimization to balance between the energy supply and the power demand. Our idea's work details will be shown in Section 3.

The main contributions of this research are summarized as follows:

- The costs of analysis power grid not only contains economics but also generator's emission, so we use a total cost (a society cost) as our optimized goal. With society development, the emission effect becomes an attentive event, hence multi-optimal goal needs to be considered, and in economic aspect, operation and maintenance are major considered. The weights of consisting total cost can be change to obtain a different optimal goal.

- Use hierarchical strategy to optimize our goal, the central level implements active power dispatch in the best way according to configuration table, the lower control level convert $P_{\text {dispatch }}$ to control value $u$ to perform $P$ out, at the same time calculate the generator's next the capacity of output according to the local weather and generator work state, then feedback the result $P_{f}$ to the central level to update configuration table.

- Embed load forecast technology into the central level, strategy implement power dispatch based on forecast result suit to the $P_{f}$

- Dispatch protocol is a form convenient to show, save and update on time.

This paper is organized as follows: Section 2 explains the problem and gives some preliminaries about machine learning technology. Section 3 states the idea's detail of this paper, and constructs optimized dispatch system model. Numerical simulations are given in Section 4. Some results and conclusions remarked are described in Section 5. The last but not the least is some acknowledgements.

\section{Problem Presents and Preliminaries}

For the further analysis, the following contents are needed.

\subsection{SVM Regression Theory in Brief [30]}

Introduce SVM's regression basic theory start with linear regression. When parameter $W$ satisfied $K K T$ the best linear regression hyperplane:

$$
f(X, W)=W^{\mathrm{T}} X+b=\sum_{i=1}^{l}\left(\alpha_{i}-\alpha_{i}^{*}\right) X_{i}^{\mathrm{T}} X+b .
$$


where $W, b$ are parameters of space, Lagrange multipliers $\alpha_{i}$ and $\alpha_{i}^{*}$.

If nonlinear regression:

$$
G=\left[\begin{array}{ccc}
G_{11} & \cdots & G_{1 l} \\
\vdots & \ddots & \vdots \\
G_{l 1} & \cdots & G_{l l}
\end{array}\right]
$$

where $G_{i j}=K\left(X_{i}, X_{j}\right)$, and the weight vector of the kernel $v_{0}=\alpha-\alpha^{*}$, therefore, regression hyperplane rewrite as follow:

$$
f(X, W)=G V_{0}+b
$$

We use SVM's regression theory to forecast electrical power load.

\subsection{Main Energy Consists of Electricity}

Generating technology including all kinds of methods, for example, traditional way hydropower plant, coal-fired generator, gas fired generator, and new energy such as Nuclear, wind power, photovoltaic generation, bioenergy power, tidal power station, and so on. Here we consider mainly most common generations technology, shown in Figure 2.

Operation costs are different for all kinds of generating technology. According to [31], we obtained the greenhouse gas emissions of different electricity. International Energy Agency (IEA) gives world energy investment outlook 2014, presented an average yearly $O \& M$, include maintenance, asset, labour, management and some important task [32]. In [33] defined operation cost includes fuel, maintenance and labour costs. All the costs and GHG are shown in following Table 1.

\subsection{Question Formulation}

We present such a goal function as below Equation (4).

$$
p_{i}=f_{i}\left(e_{i}, c_{i}, r_{i}\right)
$$

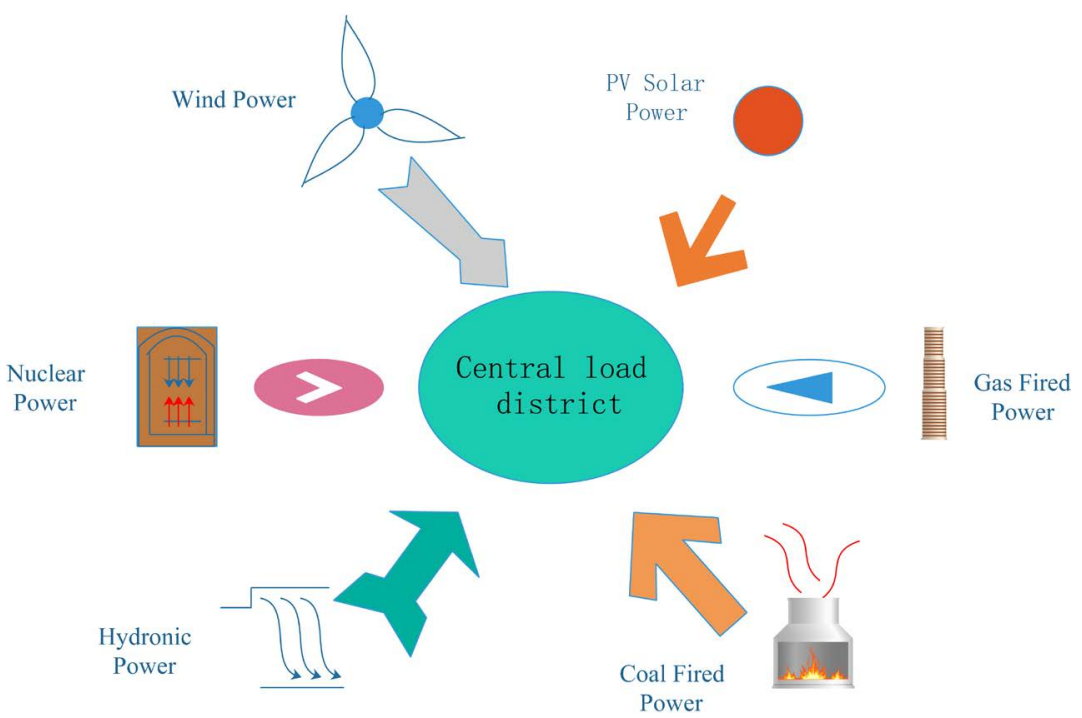

Figure 2. The main technologies of producing electric power energy. 
Table 1. Operation and maintenance cost and GHGs of main power technology.

\begin{tabular}{cccc}
\hline Technology & Operation cost [33] (US\$) & O \& M [32] (US\$) & GHG emissions [31] \\
\hline Coal Fired Power & $0.02-0.04$ & 43 & 973 \\
Gas Fired Power & $0.04-0.1$ & 20 & 450 \\
Wind Power & $<0.01$ & 46 & 23 \\
Nuclear Power & $0.02-0.05$ & 198 & 31.7 \\
Photovoltaic Solar Power & $<0.01$ & 25 & 84.8 \\
Hydro Power & $<0.01$ & 53 & 36.9 \\
\hline
\end{tabular}

where $p_{i}$ denotes dispatch power of the generator $i, f_{i}$ denotes the function on generator, $e_{i}, c_{i}, r_{i}$ denotes environment situations, generation type class and output ratio of the generator $i$ respectively. The parameter $c_{i}$ will get different consider based on our optimized purpose, $e_{i}$ is designed for environment situation, for example if its night time and daytime we should give different considering to solar energy. Here, we mainly aim to the total cost including many contents like Table 1 , so we can get $c_{i}$. And about $r_{i}$ denotes the situation of tracking the best output of a generator.

$$
\begin{aligned}
\text { Minimize: } & S_{\text {cost }}=\sum_{i=1}^{l} S_{\text {cost }}\left(P_{g}(i)\right) . \\
\text { Subject to : } & \sum_{i=1}^{l} P_{g}(i)-\sum P_{d}=0, \\
& P_{G} \in\left[0, P_{\text {gmax }}\right], \\
& P_{D} \in\left[0, P_{\text {dmax }}\right] .
\end{aligned}
$$

where $P_{G}$ denotes the total generations of all running generators in the power grid, $P_{D}$ describes the total demand of the power grid. $P_{\text {gmax }}, P_{d \max }$ denote the maximal generation and demand respectively. $S_{\text {cost }}$ is the goal cost include minimize total cost 6 and track the best output power of the generator.

\subsection{CLCT Data Obtained and Pre-Prepare}

Here we normalize the cost of different main power technology to convenient to analysis, the normal method shown in (6).

$$
\begin{aligned}
& C_{n}(i)=c_{n}(i) / \sum_{i=1}^{6} c_{n}(i), n=1,2,3, \\
& \text { Total }_{\text {cost }}=\sum_{n=1}^{3} \lambda_{n} C_{n} .
\end{aligned}
$$

where, $c_{n}(i)$ means the cost of the $n$ item of $i$ type generator, $C_{n}(i)$ denotes the normal cost of $c_{n}(i)$. Total $l_{\text {cost }}$ is the total cost of three type cost of a generator described in Table $1, \lambda_{n}$ is the coefficient of $C_{n}$, we can change it to fit different needs. For example we just keep the $\lambda$ of GHG when only consider air emission and let the others $\lambda$ equal zero.

We get the bar chart of costs about generators according to Table 1 and above 
normal algorithm of Equation (6) are shown as following Figure 3. And power system data comes from European Statistic Report

\section{https://www.entsoe.eu/data/statistics/.}

We get the power hourly load data of BG, and divide original power data into training data and testing data. Firstly, we use three days data for training and testing, then we use the head of eighteen data of a day as training data, the rest data of the day for predicting.

\section{CLCT Design Optimize Protocol}

In order to realize power dispatch in the future smart grid, we propose a hierarchy optimize protocol is shown in Figure 4. Where, the theme of scenario is the grid dispatch strategy, the agent from 1 to $n$ is generator system local controller, generator from 1 to $n$ denote the generating device. we give such as the explanation about the strategy scheme, dispatch centre dispatches $P_{W}$ to every agent, then agent converts it to control variable $u$ to control generator make it output corresponding active power Pout when the grid frequency fluctuate extra the stability range. In the feedback route, according to the work state parameter $w$ and real-time weather condition agent feedback the next generate active to dispatch centre, then the dispatch centre can give correct power dispatch configuration table.
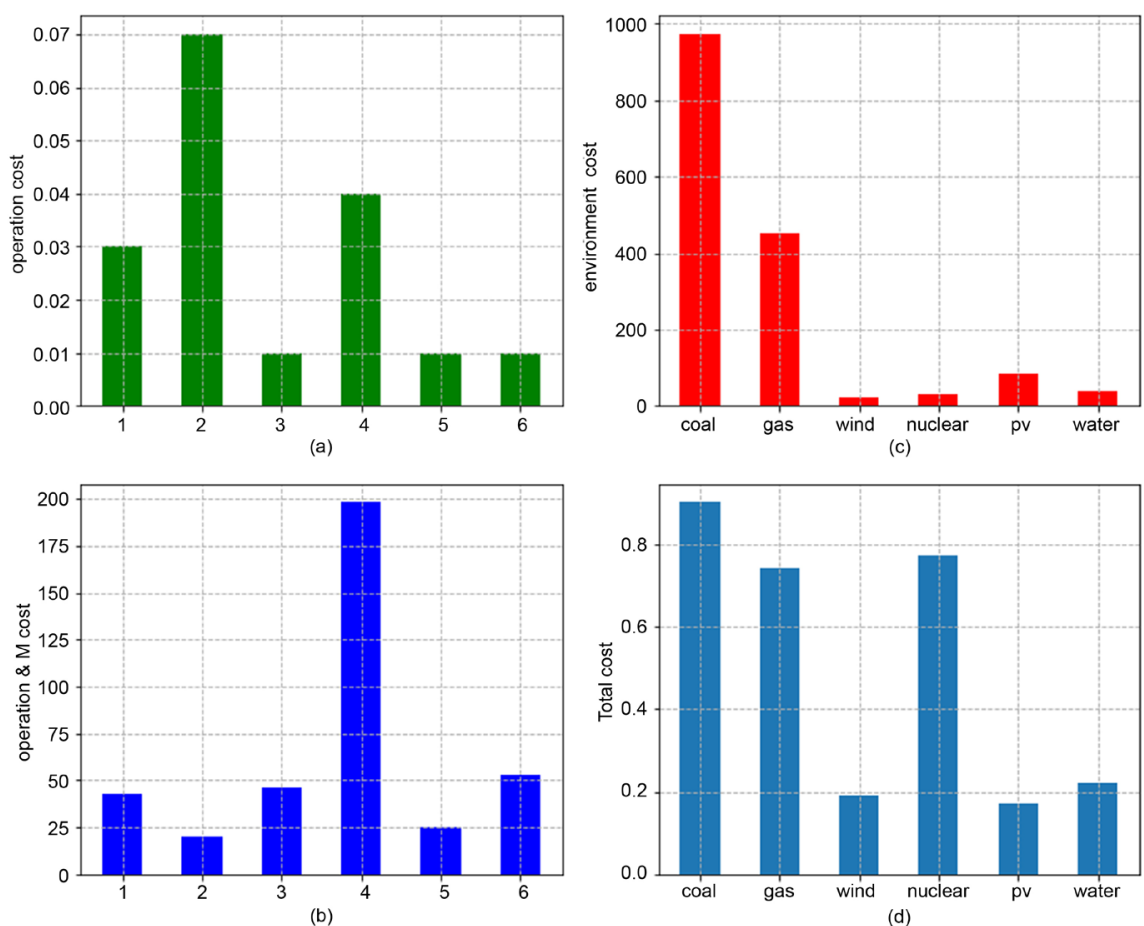

Figure 3. All kinds of costs of different type generators, (a) denotes operation cost include fuel, maintenance and labour costs; (b) denotes include maintenance, asset, labour, management and some important task costs; (c) denotes emission factors are based on the Global Emission Model for integrated systems database version 4.5 [20]; (d) denotes integrated cost of different source energy. 
In this strategy, the most important thing is to decide controlling which generators in a grid. Our scenario is shown in Figure 4, it is realized by sorting all the generators in the power grid by its cost value from smallest to the biggest, which include generator's economic cost and environment affecting cost. Then we select generators start from the first one satisfied our goal. When more one generators have the same cost we select generators according to the second-rank-factor by generator's output rating from big one to small one. This way can satisfy prior to use bigger generator output as basement load, and reduce generator's power loss. So we design the generator's work state form sorted by rank two.

Notes: the result of this algorithm is a dispatch generating configuration table according to minimize total cost, and consider Equation (4) $v_{i}$ and $c_{i}$ individually, $c_{i}$ is the first place and $v_{i}$ behind of it. We realize $c_{i}$ through the first factor sort based on total cost of the generator, realize $v_{i}$ via the second factor according to active power from bigger to smaller.

The scheme of the strategy we use the process as following to show you. First of all, we introduced the upper strategy is how to create dispatch power data. Then we introduced the lower control agent how to do it after getting the dispatch task.

Step 1: Data prepare. The parameters of generator were shown 1 normalized as Table 2, according electric power load data to forecast next power load value.

> Step 2: Design a form for output strategy and initialize it. This form is ranked by two parameters, that is generator's cost and generator's output rating individually. When first time running system, we must let the work state column of the form equal zero.

Step 3: Load forecast. We predict next some time load in the power grid use machine learning method by last period time running load data in the grid. then we got a predicted value of the next load $P_{f}$

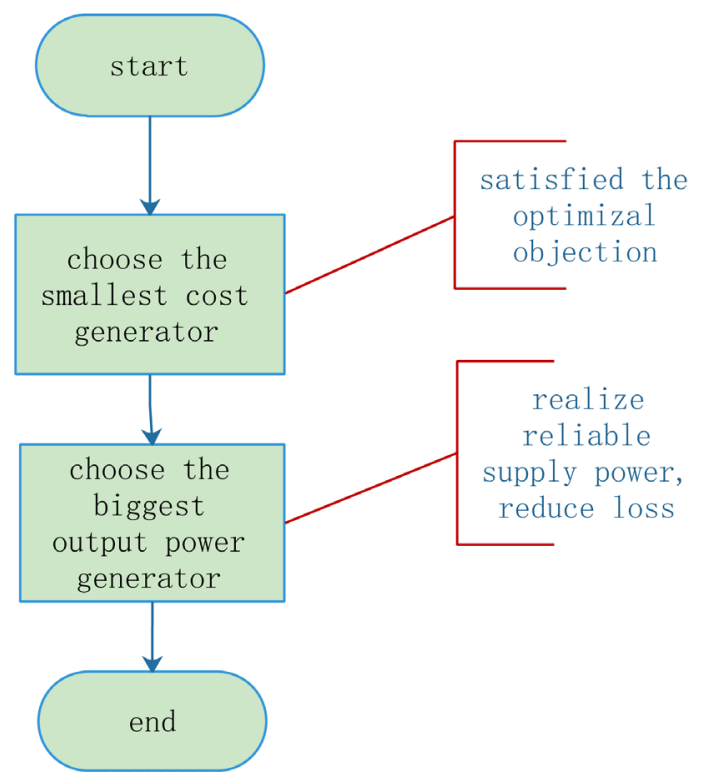

Figure 4. Schematic chart of our scenario of selection algorithm. 
Table 2. Operation and maintenance cost and GHGs of main power normalize technology.

\begin{tabular}{cccc}
\hline Technology & $\begin{array}{c}\text { normalized } \\
\text { operation cost }\end{array}$ & normalized O \& M & $\begin{array}{c}\text { normalized } \\
\text { GHG emissions }\end{array}$ \\
\hline Coal Fired Power & 0.18 & 0.11 & 0.61 \\
Gas Fired Power & 0.41 & 0.05 & 0.28 \\
Wind Power & $<0.06$ & 0.12 & 0.01 \\
Nuclear Power & 0.24 & 0.51 & 0.02 \\
Photovoltaic Solar Power & 0.06 & 0.06 & 0.05 \\
Hydro Power & 0.06 & 0.14 & 0.02 \\
\hline
\end{tabular}

Step 4: Based on $P_{f}$ implement dispatch strategy. Firstly we need to get the generators' cost from the form, then choose generators to assign active power which fits our protocol rules, about its details as shown in algorithm Figure 5.

Step 5: Ahead of step 4, we have got the dispatch table, and know the work state of all generators in a grid in the next work's situation, that's the column workstate in the form. This step we need to do some jobs on converting and control in lower agent, convert the dispatch active $P$ to control generator's variable $u$ to perform active output. The method is that when grid frequency fluctuate range extra power grid allowance trigger controller output $u$, about some this rule details refer from Figure 6 to Figure 8.

\section{CLCT Simulation}

\subsection{Load Forecast of Power Grid}

Load forecast schematic described in Figure 9, when the power grid work on $t$, we need to predict $t 2$ load use last period data. We use predict power data were taken from Bulgaria (BG) power data of March 2017, first, we give one-month load data as shown in Figure 10. In order to conveniently observe trained the SVR model use three days data, then use the last five data of first-day testing. Model accuracy is calculated use Equation (7) and the result shown in Figure 11.

$$
\begin{aligned}
\text { RMSE } & =\sqrt{1 / n \sum_{i=1}^{n}\left(y_{t}(i)-y_{r}(i)\right)^{2}}, \\
\text { MAPE } & =\operatorname{RMSE} /\left(1 / n \sum_{i=1}^{n} y_{r}(i)\right) * 100
\end{aligned}
$$

where, $y_{t}(i)$ denotes forecast value, $y_{r}(i)$ power grid real data. based on Figure 12 we got the RMSE is $54.02 \mathrm{MW}$, MAPE is $1.09 \%$, which indicates our forecast is efficient.

\subsection{CLCT Scenario of Dispatch Active Power}

Suppose BG power grid has a consisted energy as shown in Table 3. This table is the original status, so all of the work states are zero. We suppose optionally $t 1$ power grid load is $546,000 \mathrm{kw}$, after running power dispatch algorithm we can 


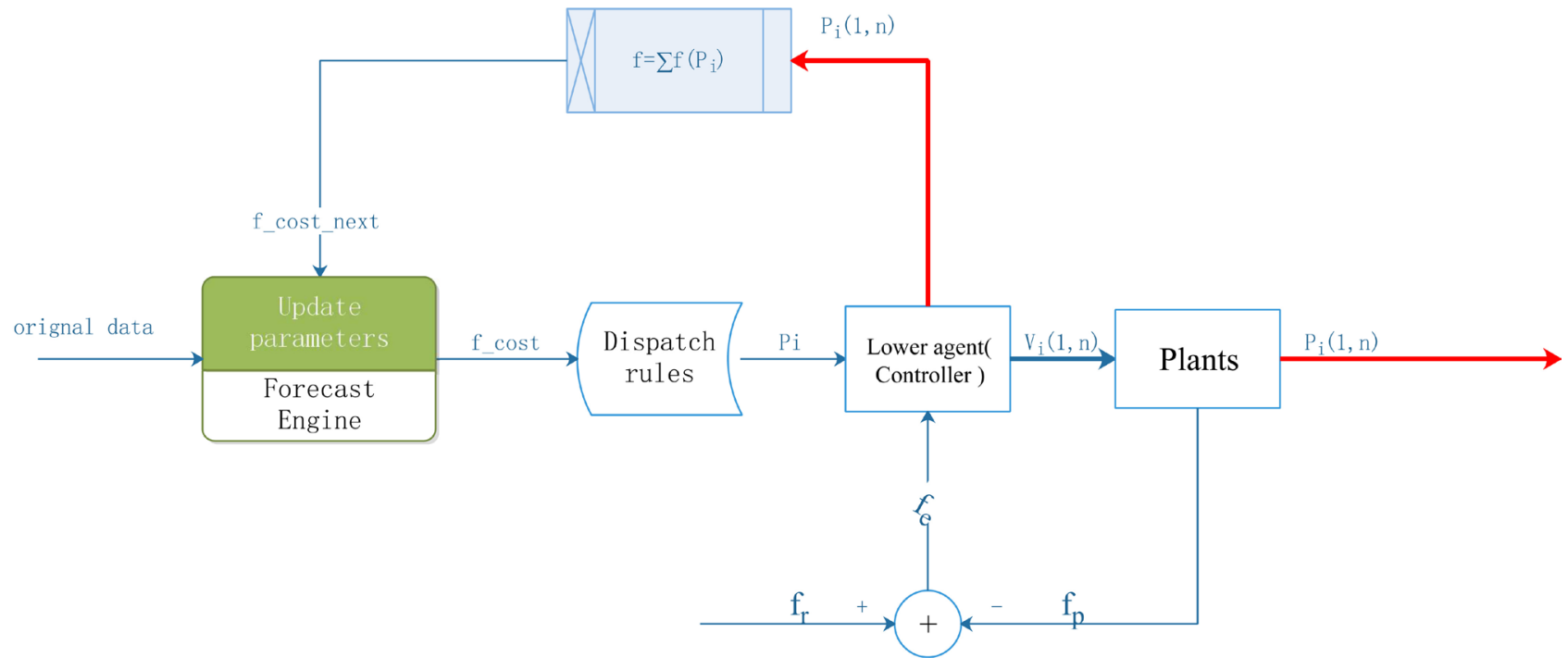

Figure 5. Strategy of our dispatch power in smart grid.

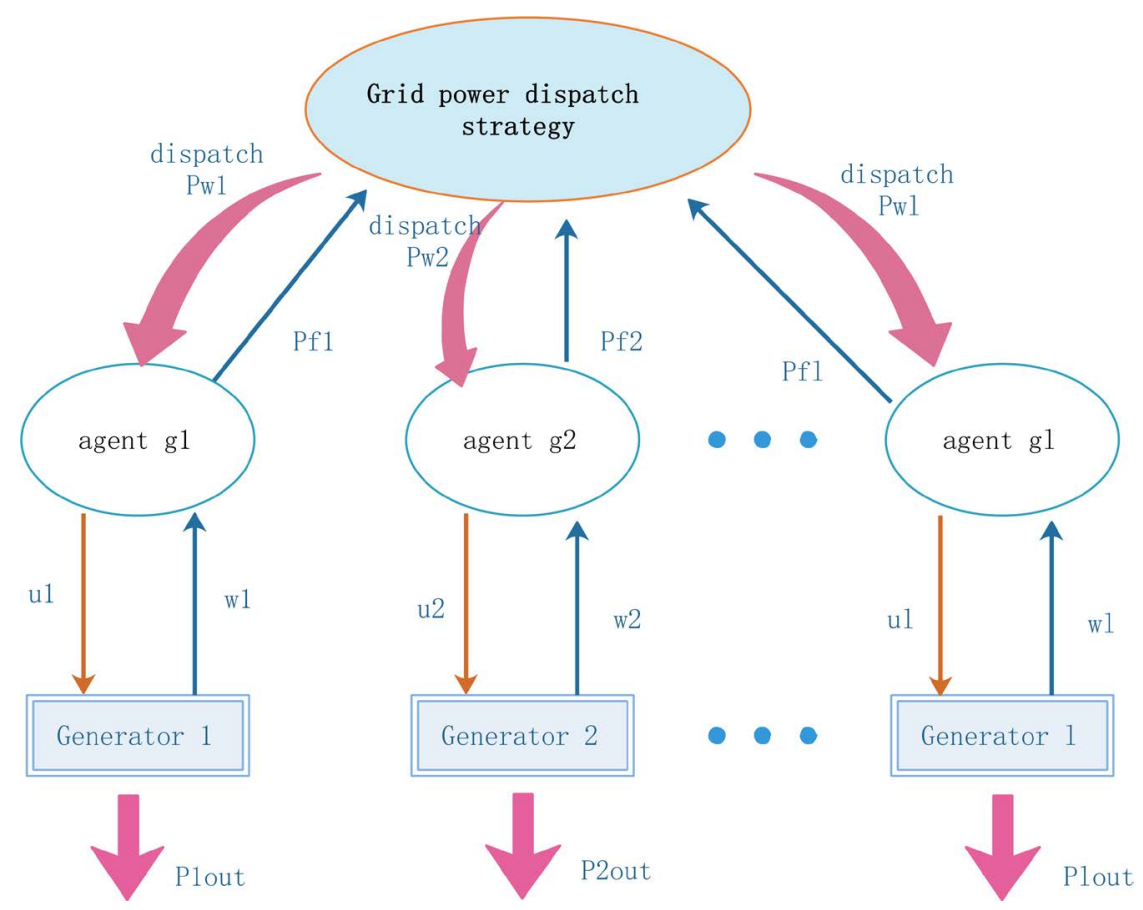

Figure 6. The chart of the power and control informs flow routing of our dispatch optimize strategy.

get the dispatch table as shown in Table 4. The figure shows that we just need G1, G3, G14, G18, G29 on working, when load is $780,000 \mathrm{kw}$ on $t 2$, the dispatch rules as Table 5, which shows just need G1, G3, G9, G11, G24, G26, G14, G18, G29 are working on rated power state.

Simulation results show that optimized algorithm of dispatch is convenient to realize by second rank sorting, and all of the worked generators are working on rated power, that's means it working on the highest efficient except only the last one. 


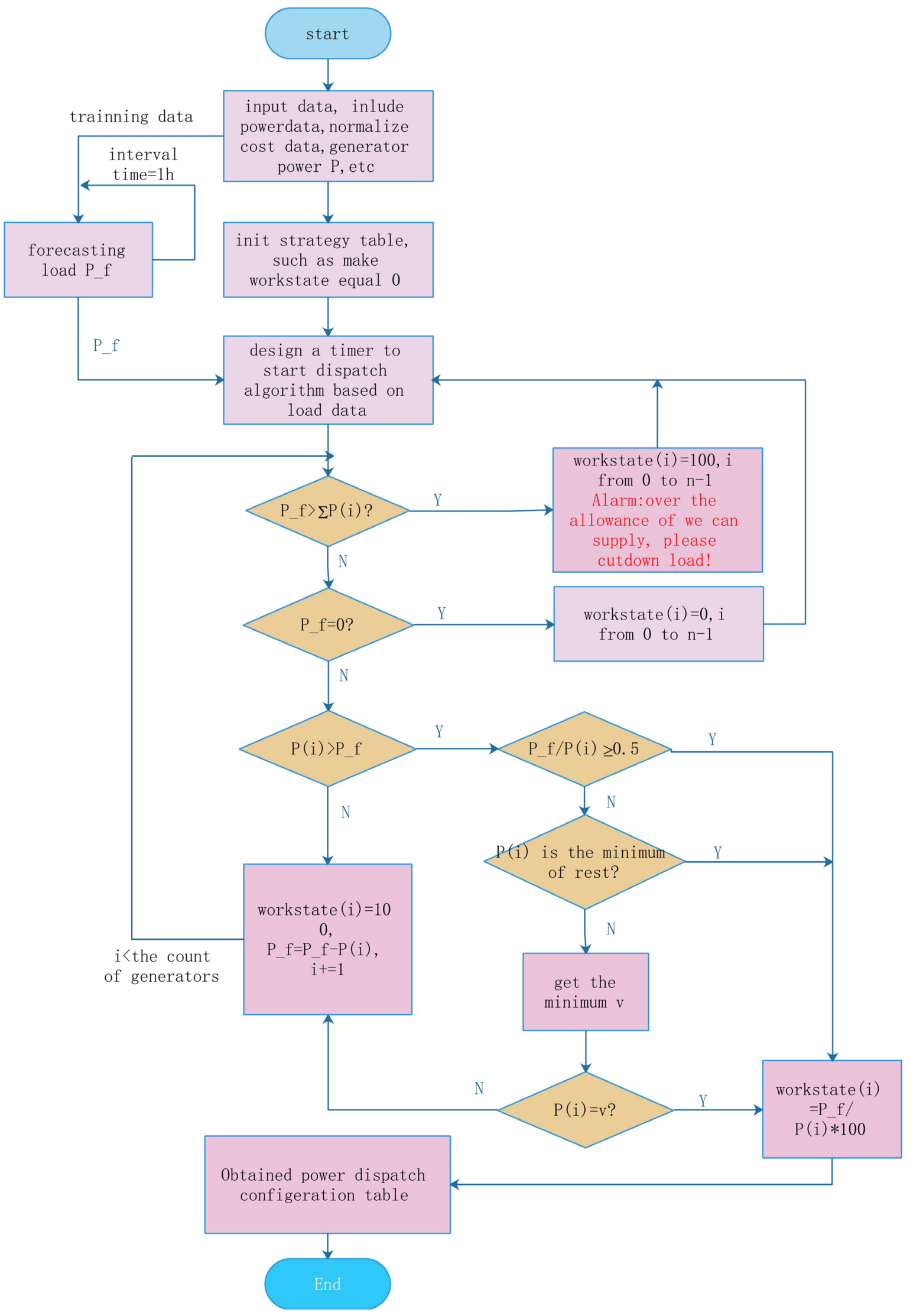

Figure 7. The flowchart of power grid active power dispatch algorithm of central level. 


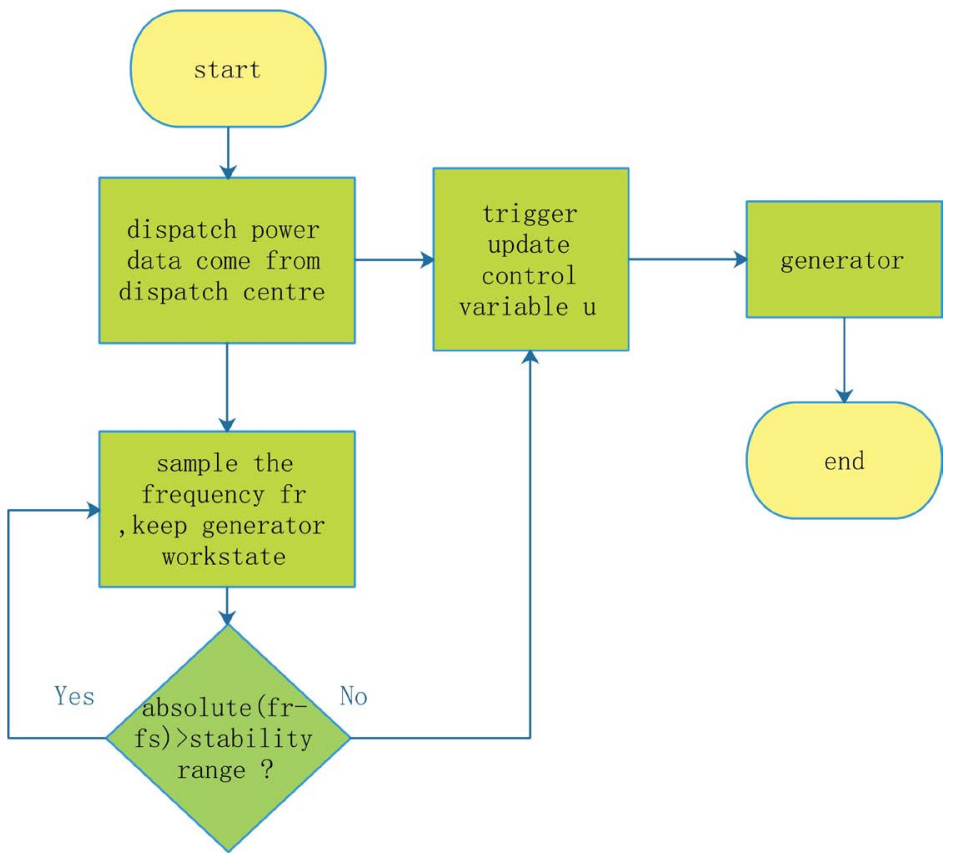

Figure 8. The flowchart of local agent control algorithm. $f s$ is the standard frequency of power grid, and dispatch come from strategy centre.

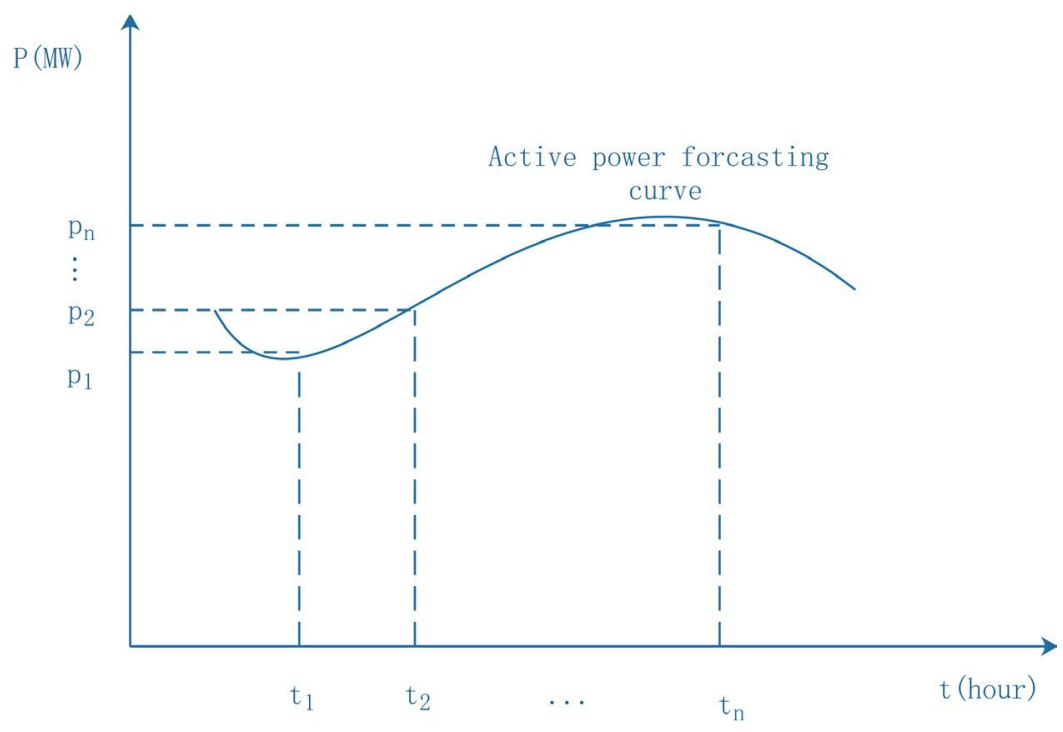

Figure 9. Forecast scheme describe.

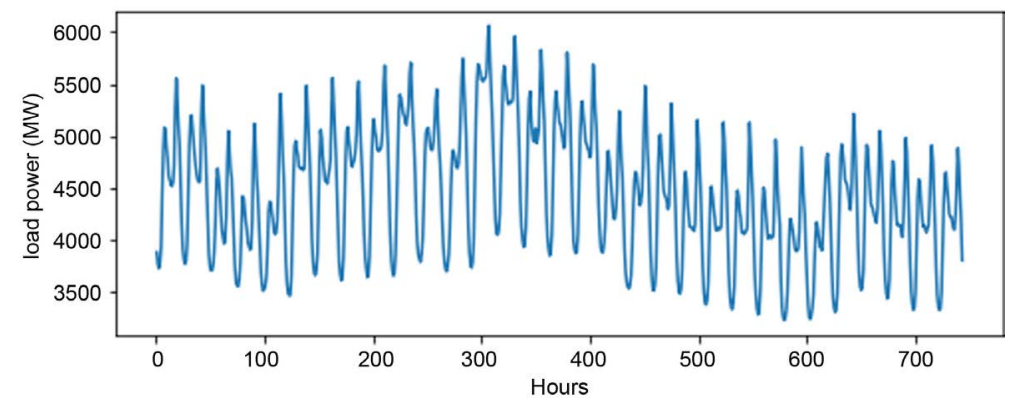

Figure 10. A month power actually load in BG. 


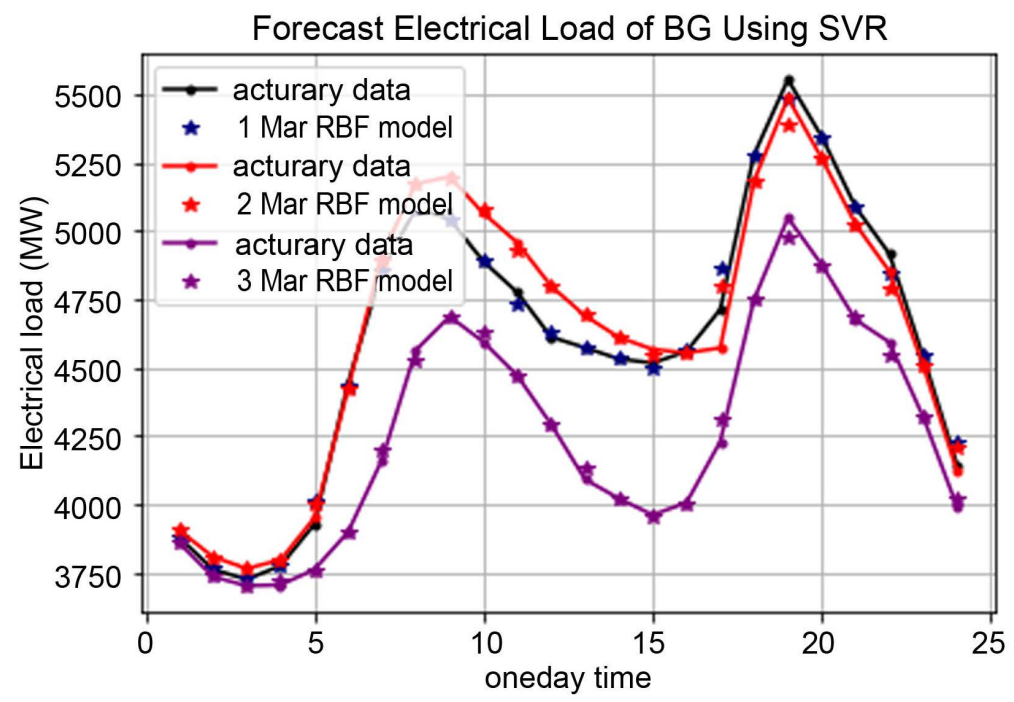

Figure 11. Power load forecast in BG using SVR. (Note the numbers of axis $\mathrm{x}$ such as " 20 " that's mean the time from 19 clock to 20.)

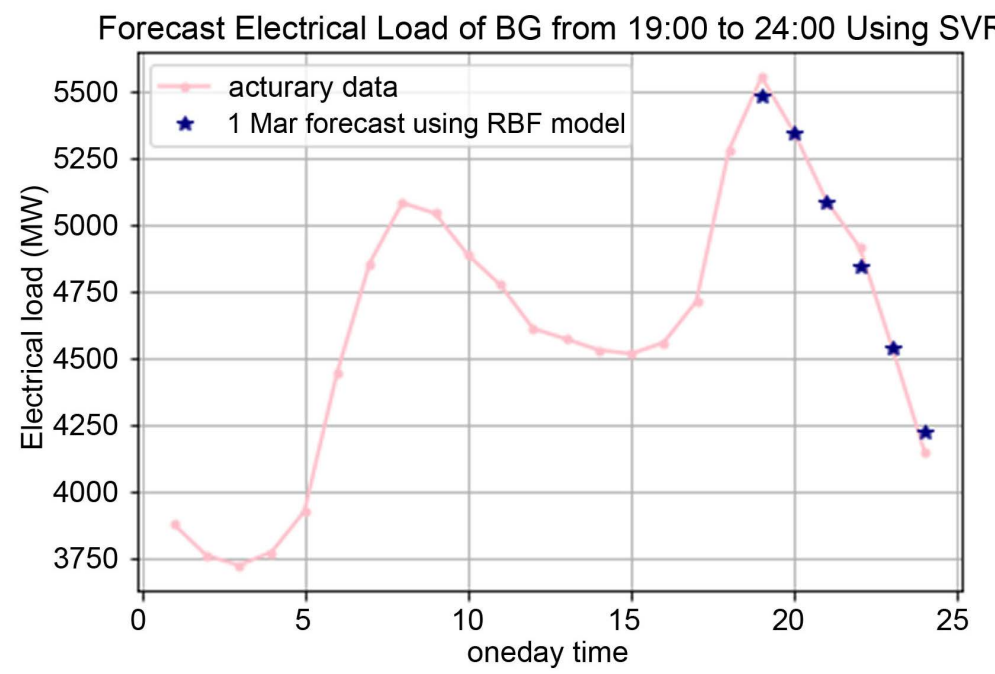

Figure 12. Forecast chart, use the data from 0:00 to 18:00 as train set and the rest of a day as test to forecast.

Table 3. A power grid energy consists of multi technology type.

\begin{tabular}{cccccc}
\hline Id & Class & Power_E $(\mathrm{kw})$ & Workstate & Distance $(\mathrm{km})$ & Total_cost \\
\hline G1 & wind & 500,000 & 0 & 30 & 0.19 \\
G2 & water & 700,000 & 0 & 150 & 0.22 \\
G3 & pv & 50,000 & 0 & 10 & 0.17 \\
G4 & gas & 5000 & 0 & 10 & 0.74 \\
G5 & nuclear & 550,000 & 0 & 200 & 0.77 \\
G6 & coal & 60,000 & 0 & 15 & 0.22 \\
G7 & water & 33,000 & 0 & 50 & 0.22 \\
G8 & coal & 60,000 & 0 & 15 & 0.9 \\
G9 & water & 50,000 & 0 & 80 & 0.22 \\
\hline
\end{tabular}




\section{Continued}

\begin{tabular}{|c|c|c|c|c|c|}
\hline G10 & water & 1000 & 0 & 50 & 0.22 \\
\hline G11 & wind & 10000 & 0 & 30 & 0.19 \\
\hline G12 & coal & 800,000 & 0 & 60 & 0.9 \\
\hline G13 & coal & 30,000 & 0 & 30 & 0.9 \\
\hline G14 & $\mathrm{pv}$ & 30,000 & 0 & 5 & 0.17 \\
\hline G15 & gas & 20,000 & 0 & 10 & 0.74 \\
\hline G16 & wind & 500,000 & 0 & 30 & 0.19 \\
\hline G17 & water & 700,000 & 0 & 150 & 0.22 \\
\hline G18 & $\mathrm{pv}$ & 50,000 & 0 & 10 & 0.17 \\
\hline G19 & gas & 5000 & 0 & 10 & 0.74 \\
\hline G20 & nuclear & 550,000 & 0 & 200 & 0.77 \\
\hline G21 & coal & 100,000 & 0 & 20 & 0.9 \\
\hline G22 & water & 33,000 & 0 & 50 & 0.22 \\
\hline G23 & coal & 60,000 & 0 & 15 & 0.9 \\
\hline G24 & water & 50,000 & 0 & 80 & 0.22 \\
\hline G25 & water & 1000 & 0 & 50 & 0.22 \\
\hline G26 & wind & 10,000 & 0 & 30 & 0.19 \\
\hline G27 & coal & 800,000 & 0 & 60 & 0.9 \\
\hline G28 & coal & 30,000 & 0 & 30 & 0.9 \\
\hline G29 & $\mathrm{pv}$ & 30,000 & 0 & 5 & 0.17 \\
\hline G30 & gas & 20,000 & 0 & 10 & 0.74 \\
\hline
\end{tabular}

Table 4. Dispatch result when forecasting value is $546,000 \mathrm{kw}$.

\begin{tabular}{ccccccc}
\hline No. & Id & Class & Power_E $(\mathrm{kw})$ & Workstate & Distance $(\mathrm{km})$ & Total_cost \\
\hline 2 & G3 & PV & 50,000 & 100 & 10 & 0.17 \\
17 & G18 & PV & 50,000 & 100 & 10 & 0.17 \\
13 & G14 & PV & 30,000 & 100 & 5 & 0.17 \\
28 & G29 & PV & 30,000 & 100 & 5 & 0.17 \\
0 & G1 & Wind & 500,000 & 77 & 30 & 0.19 \\
\hline
\end{tabular}

Table 5. Dispatch result when forecasting value is 780,000 kw.

\begin{tabular}{ccccccc}
\hline No. & Id & Class & Power_E $(\mathrm{kw})$ & Workstate & Distance $(\mathrm{km})$ & Total_cost \\
\hline 2 & G3 & PV & 50,000 & 100 & 10 & 0.17 \\
17 & G18 & PV & 50,000 & 100 & 10 & 0.17 \\
13 & G14 & PV & 30,000 & 100 & 5 & 0.17 \\
28 & G29 & PV & 30,000 & 100 & 5 & 0.17 \\
0 & G1 & Wind & 500,000 & 100 & 30 & 0.19 \\
10 & G11 & Wind & 10,000 & 100 & 30 & 0.19 \\
25 & G26 & Wind & 10,000 & 100 & 30 & 0.19 \\
8 & G9 & Water & 50,000 & 100 & 80 & 0.22 \\
23 & G24 & Water & 50,000 & 100 & 80 & 0.22 \\
\hline
\end{tabular}


Notes: Hypothesis we have got a power grid energy consists of Table 3, that is the original situation, the column of power_E will be changed. When the weather changed the lower agent will change the generator's power_E, for example, the PV's power_E is different between sunny day and cloudy day.

\section{Conclusions and Future Works}

In this paper, we address a new approach to realize power configuration in the smart grid, and make dispatch strategy reflect in a configuration table based on operation cost and environment effect cost. This method consists of two-level parts, dispatch configuration central and lower control agent of the generator. In the central, we mainly did these things: dispatching optimized calculation, converting it into configuration table, and updating it. The lower control agent aims to realize control generators according to central and feedback generators' new best working power based on the situation. In the central level, use machine learning to predict power load, and address the best-optimized cost function to adapt to the different control strategies. Then we do dispatch based on the table, which can be updated online based on environmental conditions. The signs of triggering controller are both active power and system $f$. The lower control agent carries out the generator to track $f$ along with the best optimized cost function. In the numerical simulation, we used BG grid load as forecast data to train machining learning model, gave two predicted load data at random, and took them as the cases. We obtained good simulation results, right dispatch configuration table, and the table has been updated online. So, this scenario makes optimized dispatch algorithm more convenient to realize and effective.

In the future work, we will keep on optimizing strategy research, consider more situations when designing cost function, and try our best to get a real large-scale power grid, which includes more generated technology.

\section{Acknowledgements}

This research was financially supported by the National Science Foundation (No. 41804148), the Education Department of Sichuan Province Foundation (No. 18ZB0273) and Leshan Science and Technology Bureau Foundation (No. 15NZD100), China. The authors would like to thank them. The power data used in this research comes from Open Power System Data:

http://www.open-power-system-data.org/, so we also thank Open Power System Data group open data.

\section{Conflicts of Interest}

The authors declare no conflicts of interest regarding the publication of this paper.

\section{References}

[1] Gharavi, B.H. and Ieee, F. (2011) Smart Grid: The Electric Energy System of the 
Future. Proceedings of the IEEE, 99, 917-921.

https://doi.org/10.1109/JPROC.2011.2124210

[2] Tuballa, M.L. and Abundo, M.L. (2016) A Review of the Development of Smart Grid Technologies. Renewable and Sustainable Energy Reviews, 59, 710-725.

https://doi.org/10.1016/j.rser.2016.01.011

[3] Fang, X., Misra, S., Xue, G. and Yang, D. (2012) Smart Grid the New and Improved Power Grid: A Survey. IEEE Communications Surveys \& Tutorials, 14, 944-980. https://doi.org/10.1109/SURV.2011.101911.00087

[4] Dusparic, I., Harris, C., Marinescu, A., Cahill, V. and Clarke, S. (2013) Multi-Agent Residential Demand Response Based on Load Forecasting. in Technologies for Sustainability. 1 st IEEE Conference on SusTech, October 2013, 90-96. https://doi.org/10.1109/SusTech.2013.6617303

[5] Wen, Z., O’Neill, D. and Maei, H.R. (2014) Optimal Demand Response Using Device Based Reinforcement Learning. 1-23. http://arxiv.org/abs/1401.1549

[6] Khezeli, K. and Bitar, E. (2016) Risk-Sensitive Learning and Pricing for Demand Response. 1-11. http://arxiv.org/abs/1611.07098

[7] Capovilla, C.E., Casella, I.R.S., Sguarezi Filho, A.J., Azcue-Puma, J.L., Jacomini, R.V. and Ruppert, E. (2014) A Wind Energy Generator for Smart Grid Applications Using Wireless-Coded Neuro-Fuzzy Power Control. Computers and Mathematics with Applications, 68, 2112-2123. https://doi.org/10.1016/j.camwa.2013.06.030

[8] Gandhi, P.R. and Joshi, S.K. (2014) Smart Control Techniques for Design of TCSC and PSS for Stability Enhancement of Dynamical Power System. Applied Soft Computing Journal, 24, 654-668. https://doi.org/10.1016/j.asoc.2014.08.017

[9] Motevasel, M. and Seifi, A.R. (2014) Expert Energy Management of a Micro-Grid Considering Wind Energy Uncertainty. Energy Conversion and Management, 83, 58-72. https://doi.org/10.1016/j.enconman.2014.03.022

[10] Ata, R. (2015) Artificial Neural Networks Applications in Wind Energy Systems: A Review. Renewable and Sustainable Energy Reviews, 49, 534-562. https://doi.org/10.1016/j.rser.2015.04.166

[11] Jayawardene, I. and Venayagamoorthy, G.K. (2015) Comparison of Adaptive Neuro-Fuzzy Inference Systems and Echo State Networks for PV Power Prediction. Procedia Computer Science, 53, 92-102. https://doi.org/10.1016/j.procs.2015.07.283

[12] Ni, Z., Tang, Y., Sui, X., He, H. and Wen, J. (2016) An Adaptive Neuro-Control Approach for Multi-Machine Power Systems. International Journal of Electrical Power and Energy Systems, 75, 108-116. https://doi.org/10.1016/j.ijepes.2015.08.012

[13] Koohi-Kamal, S., Rahim, N., Mokhlis, H. and Tyagi, V. (2016) Photovoltaic Electricity Generator Dynamic Modeling Methods for Smart Grid Applications: A Review. Renewable and Sustainable Energy Reviews, 57, 131-172. http://linkinghub.elsevier.com/retrieve/pii/S1364032115015208 https://doi.org/10.1016/j.rser.2015.12.137

[14] De Santis, E., Rizzi, A. and Sadeghian, A. (2016) A Hierarchical Genetic Optimization of a Fuzzy Logic System for Flow Control in Micro Grids. Applied Soft Computing Journal. http://arxiv.org/abs/1604.04789

[15] Mahmoud, M.S., Alyazidi, N.M. and Abouheaf, M.I. (2017) Adaptive Intelligent Techniques for Microgrid Control Systems: A Survey. International Journal of Electrical Power \& Energy Systems, 90, 292-305.

http://linkinghub.elsevier.com/retrieve/pii/S0142061516325042

https://doi.org/10.1016/j.ijepes.2017.02.008 
[16] Nadjemi, O., Nacer, T., Hamidat, A. and Salhi, H. (2017) Optimal Hybrid PV/Wind Energy System Sizing: Application of Cuckoo Search Algorithm for Algerian Dairy Farms. Renewable and Sustainable Energy Reviews, 70, 1352-1365. https://doi.org/10.1016/j.rser.2016.12.038

[17] Almonacid, F., Fernandez, E.F., Mellit, A. and Kalogirou, S. (2017) Review of Techniques Based on Artificial Neural Networks for the Electrical Characterization of Concentrator Photovoltaic Technology. Renewable and Sustainable Energy Reviews, 75, 938-953. https://doi.org/10.1016/j.rser.2016.11.075

[18] Theo, W.L., Lim, J.S., Ho, W.S., Hashim, H. and Lee, C.T. (2017) Review of Distributed Generation (DG) System Planning and Optimisation Techniques: Comparison of Numerical and Mathematical Modelling Methods. Renewable and Sustainable Energy Reviews, 67, 531-573. https://doi.org/10.1016/j.rser.2016.09.063

[19] Indragandhi, V., Subramaniyaswamy, V. and Logesh, R. (2017) Resources, Configurations, and Soft Computing Techniques for Power Management and Control of PV/Wind Hybrid System. Renewable and Sustainable Energy Reviews, 69, 129-143. https://doi.org/10.1016/j.rser.2016.11.209

[20] Zhao, C., He, J., Cheng, P. and Chen, J. (2016) Consensus-Based Energy Management in Smart Grid with Transmission Losses and Directed Communication. IEEE Transactions on Smart Grid, 8, 2049-2061. https://doi.org/10.1109/TSG.2015.2513772

[21] Boroojeni, K.G., Amini, M.H., Iyengar, S.S., Rahmani, M. and Pardalos, P.M. (2017) An Economic Dispatch Algorithm for Congestion Management of Smart Power Networks. Energy Systems, 8, 643-667. https://doi.org/10.1007/s12667-016-0224-6 http://link.springer.com/10.1007/s12667-016-0224-6

[22] Craparo, E., Karatas, M. and Singham, D.I. (2017) A Robust Optimization Approach to Hybrid Microgrid Operation Using Ensemble Weather Forecasts. Applied Energy, 201, 135-147. https://doi.org/10.1016/j.apenergy.2017.05.068

[23] Lu, P., Zhao, J., Yao, J. and Yang, S. (2017) A Decentralized Approach for Frequency Control and Economic Dispatch in Smart Grids. IEEE Journal on Emerging and Selected Topics in Circuits and Systems, 7, 447-458. https://doi.org/10.1109/JETCAS.2017.2708900

[24] Samimi, A., Nikzad, M. and Siano, P. (2017) Scenario-Based Stochastic Framework for Coupled Active and Reactive Power Market in Smart Distribution Systems with Demand Response Programs. Renewable Energy, 109, 22-40. https://doi.org/10.1016/j.renene.2017.03.010

[25] De Azevedo, R., Cintuglu, M.H., Ma, T. and Mohammed, O.A. (2017) Multiagent-Based Optimal Microgrid Control Using Fully Distributed Diffusion Strategy. IEEE Transactions on Smart Grid, 8, 1997-2008. https://doi.org/10.1109/TSG.2016.2587741

[26] Zeng, B., Wu, G., Wang, J., Zhang, J. and Zeng, M. (2017) Impact of Behavior-Driven Demand Response on Supply Adequacy in Smart Distribution Systems. Applied Energy, 202, 125-137. https://doi.org/10.1016/j.apenergy.2017.05.098

[27] Hu, X., Zhou, H., Liu, Z., Yu, X. and Li, C. (2017) Hierarchical Distributed Scheme for Demand Estimation and Power Reallocation in a Future Power Grid. IEEE Transactions on Industrial Informatics, 13, 2279-2290. http://ieeexplore.ieee.org/document/7857788 https://doi.org/10.1109/TII.2017.2670065

[28] Yu, W., Li, C., Yu, X., Wen, G. and Lu, J. (2018) Economic Power Dispatch in Smart Grids: A Framework for Distributed Optimization and Consensus Dynamics. 
Science China Information Sciences, 61, Article ID: 012204.

https://doi.org/10.1007/s11432-016-9114-y

[29] Meng, W. and Wang, X. (2017) Distributed Energy Management in Smart Grid with Wind Power and Temporally Coupled Constraints. IEEE Transactions on Industrial Electronics, 64, 6052-6062. http://ieeexplore.ieee.org/document/7878614 https://doi.org/10.1109/TIE.2017.2682001

[30] Kecman, V. (2005) Support Vector Machines: An Introduction. In: Wang, L.P., Ed., Support Vector Machines. Theory and Applications, Springer, Berlin, 1-47. https://doi.org/10.1007/10984697_1

[31] Shih, Y.-H. and Tseng, C.-H. (2014) Cost-Benefit Analysis of Sustainable Energy Development Using Life-Cycle Co-Benefits Assessment and the System Dynamics Approach. Applied Energy, 119, 57-66.

https://doi.org/10.1016/j.apenergy.2013.12.031

http://linkinghub.elsevier.com/retrieve/pii/S0306261913010301

[32] IEA (2014) Power Plant O\&M: How Does the Industry Stack up on Cost? Power Technology. Tech. Rep.

http://www.power-technology.com/features/featurepower-plant-om-how-does-theindustry-stack-up-on-cost-4417756

[33] I. C. C. Centre (2016) Operating Ratio and Cost of Coal Power Generation. Tech. Rep., 42. 